\title{
The role of PET scans in the management of Cholangiocarcinoma and Gallbladder Cancer: A systematic review for surgeons
}

\author{
Sanket Srinivasa, Bernard McEntee, J onathan B. Koea \\ Department of Surgery, North Shore Hospital, Takapuna, Auckland, New Zealand. \\ Correspondence: Jonathan B. Koea. Address: Department of Surgery, North Shore Hospital, Private Bag 93503, \\ Takapuna, Auckland, New Zealand. Email: jonathan.koea@waitematadhb.govt.nz \\ Received: J une 30, 2014 \\ Accepted: August 12, 2014 \\ Online Published: September 2, 2014 \\ DOI : $10.5430 /$ ijdi.v2n1p1 \\ URL: http://dx.doi.org/10.5430/ijdi.v2n1p1
}

\begin{abstract}
Introduction: Radical surgical resection is the only potentially curative therapy for cancers arising in biliary epithelia (gallbladder cancer and cholangiocarcinoma). Accurate radiological staging is crucial and the role of positron emission tomography-CT (PET-CT) scanning in this regard is undefined.

Methods: A systematic review of the literature was carried out to define the accuracy of PET-CT in patients with cholangiocarcinoma or gallbladder cancer in defining metastatic disease in portal, and extra-portal lymph nodes, the presence of intra-hepatic satellite lesions, extra-abdominal metastases and recurrent disease in patients following treatment. Results were displayed in a narrative format with no meta-analysis feasible.

Results \& Conclusions: Four hundred and sixty eight records were screened, 49 were assessed and 12 studies were included in the systematic review. PET-CT has a low sensitivity for regional lymph node metastases from cholangiocarcinoma and gallbladder cancer but a high specificity. In one study, PET-CT appears to be effective in assessing all nodal basins related to the liver, bile ducts and gallbladder. PET-CT has no role in the assessment of hepatic satellite lesions but is useful in defining extra-abdominal metastatic disease and recurrent disease and to assess specific areas of concern defined on CT scan and/or magnetic resonance scan.
\end{abstract}

\section{Keywords}

PET scan, Staging, Cholangiocarcinoma, Gallbladder cancer

\section{I ntroduction}

Cancers of biliary epithelia can occur at a number of sites presenting either in the gallbladder as a carcinoma, as an intra-hepatic cholangiocarcinoma or an extra-hepatic cholangiocarcinoma occurring in the upper, middle or lower thirds of the extrahepatic bile ducts. Currently, the only potentially curative treatment available for gallbladder carcinoma and cholangiocarcinoma in any site is radical surgical resection encompassing the primary tumour and regional lymph nodes ${ }^{[1]}$. This surgery is often technically challenging and is associated with significant morbidity and mortality since extended hepatic resections or pancreaticoduodenectomy can be required. In addition, most patients presenting with these tumours are elderly and in the 6th or 7 th decades of life and have significant co-morbidities ${ }^{[1,2]}$. Consequently, accurate 
clinical and radiological staging is critical in selecting those patients in whom surgery can be undertaken with the expectation of cure.

Radiological staging is important in defining the presence of metastatic disease, presenting either as satellite lesions within the liver (which are markers of vascular invasion) or metastases at more distant sites such as lung or bone. In addition, staging is particularly important in defining the presence and extent of metastatic involvement of regional lymph nodes since involvement of lymph nodes at the porta does not necessarily mandate against surgical resection but metastases to more distant nodes such as in the celiac, retropancreatic and para-aortic chains almost certainly do ${ }^{[1,2]}$. Finally recent advances in systemic therapy for biliary carcinomas have also highlighted the importance of accurate pre-operative staging in selecting patients with unresectable tumours that could be effectively treated with chemotherapy and those with borderline tumours who might benefit from neoadjuvant therapy with restaging prior to considering surgical resection ${ }^{[3]}$.

Alongside conventional imaging with ultrasound, computed tomographic (CT) scans and magnetic resonance (MRI) scans, positron emission tomography-CT scanning (PET-CT) is being increasingly used to guide surgical management in patients with primary or recurrent carcinomas of the gallbladder and biliary tract. Previous studies have indicated a possible benefit in detecting lymphadenopathy and metastases in the presence of a normal CT scan ${ }^{[4]}$. Its role in this capacity is, however, undefined. There is minimal recent evidence on this subject and previous papers have often been written from a radiological rather than a surgical perspective ${ }^{[4]}$.

Thus, the aim of this study was to conduct a systematic narrative review of recent evidence pertaining to the use of PET-CT for patients with primary or recurrent gallbladder carcinoma or cholangiocarcinoma (either intra-hepatic or extrahepatic location) emphasizing the current evidence as it relates to staging required prior to considering patients for radical surgical resection. There was a specific focus on the proportion of patients whose management would differ from the additional information gained from a PET-CT rather than on statistical measures of precision and accuracy alone. A secondary aim of the study was to draw conclusions about the strengths of PET-CT in staging the surgically important subtypes of these cancers (e.g. extra-hepatic vs. intra-hepatic cholangiocarcinoma). This investigation was undertaken to answer the following questions.

1) In patients presenting with biliary (intra-hepatic cholangiocarcinoma, extrahepatic cholangiocarcinoma and gallbladder) cancer how effective is PET-CT in defining metastases to regional lymph nodes (portal, retropancreatic, celiac and para-aortic basins).

2) In patients with biliary cancer how effective is PET-CT in defining the presence of intra-hepatic metastases (satellite lesions).

3) In patients with biliary cancer how effective is PET-CT is defining metastatic disease in extra-abdominal sites.

4) In patients with biliary cancer who had already undergone potentially curative resection how effective is PET-CT in defining recurrent disease within the tumour bed, regional lymph nodes or at distant sites.

\section{Methods}

\subsection{Systematic literature search}

The following terms were used to devise a search: Gallbladder cancer; Gallbladder neoplasms; Cholangiocarcinoma; bile duct cancer; PET; positron emission tomography. The search was run independently by two authors (SS, BM) according to the validated methods of the PRISMA statement ${ }^{[5]}$. There were no restrictions on language. The databases examined were MEDLINE (1966-2013), Cochrane Central Register of Controlled Trials, EMBASE (1947-2013), PubMed and Web of Science. The reference lists of all included papers, as well as related review articles, were manually searched to identify further relevant studies. 


\subsection{Study selection}

Published studies that evaluated the use of PET-CT in patients with primary or recurrent cholangiocarcinoma or gallbladder cancer were included. Exclusion criteria were: Studies published prior to 2008; review articles; case reports; articles not specific to PET-CT and cholangiocarcinoma/ gallbladder cancer. Papers were scrutinised for inclusion independently by two authors (SS, BM) with disagreement resolved by consultation with the senior author (JBK) if consensus could not be reached.

\subsection{Outcomes assessed}

Study characteristics were noted and the outcomes of interest were the detection of primary disease, satellite lesions, nodal disease and metastases. Recurrent disease was also examined as appropriate. These outcomes were noted for gallbladder cancer and cholangiocarcinoma separately with further stratification by anatomic site for the latter. When further details were not available, the results were noted for cholangiocarcinoma or biliary cancer alone. Statistical measures of accuracy were noted by reporting sensitivity and specificity as well as the eventual proportion of patients whose management was changed by the use of PET-CT.

\section{Results}

The literature search yielded 12 papers ${ }^{[6-17]}$ for inclusion in the study as outlined in the PRISMA statement of search results (see Figure 1). The study characteristics for all included papers are summarised in Table 1 and the results as they pertain to the four clinical questions driving this review are summarized in Table 2.

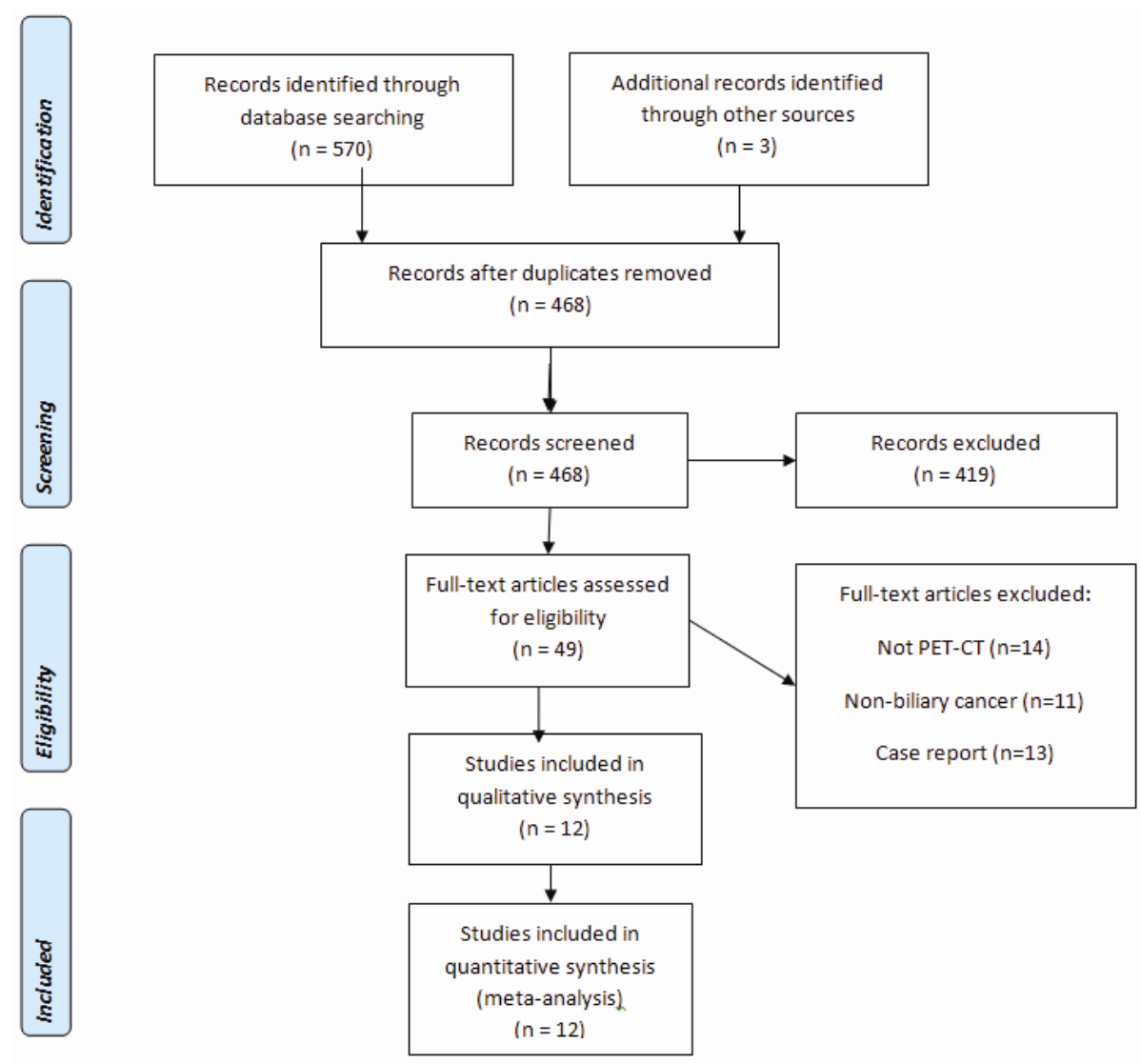

Figure 1. PRISMA Statement of search results 
Table 1. Summary of study characteristics

\begin{tabular}{|c|c|c|c|c|c|}
\hline Author & Year & No. of Patients & Study design & Malignancy & Diagnostic criteria \\
\hline Butte ${ }^{[6]}$ & 2009 & 32 & Pro & GBC & SQ \\
\hline Corvera $^{[7]}$ & 2008 & 126 & Retro & $\begin{array}{l}\text { GBC/CC } \\
\text { (Intrahepatic/ } \\
\text { Extrahepatic) }\end{array}$ & SQ \\
\hline Furukawa ${ }^{[8]}$ & 2008 & 72 & Retro & $\begin{array}{l}\text { GBC/ } \\
\text { (Extrahepatic) }\end{array}$ & Visual \\
\hline $\operatorname{Kim}^{[9]}$ & 2008 & 123 & Pro & $\begin{array}{l}\text { CC (Intrahepatic/ } \\
\text { Extrahepatic/ Hilar) }\end{array}$ & SQ/ Visual \\
\hline Kitajima ${ }^{[10]}$ & 2009 & 50 & Retro & GBC/CC & Visual \\
\hline Kumar $^{[11]}$ & 2012 & 49 & Retro & GBC & Visual \\
\hline Lee $^{[13]}$ & 2010 & 99 & Retro & $\begin{array}{l}\text { CC (Intrahepatic/ } \\
\text { Extrahepatic }\end{array}$ & SQ/ Visual \\
\hline Lee ${ }^{[12]}$ & 2011 & 50 & Retro & $\begin{array}{l}\text { GBC/CC } \\
\text { (Intrahepatic/ } \\
\text { Extrahepatic }\end{array}$ & SQ/ Visual \\
\hline $\mathrm{Li}^{[14]}$ & 2008 & 17 & Pro & CC (Hilar) & SQ/ Visual \\
\hline Ruys ${ }^{[15]}$ & 2011 & 30 & Retro & CC (Hilar) & $\mathrm{SQ} /$ Visual \\
\hline Seo ${ }^{[16]}$ & 2008 & 27 & Retro & CC (Intrahepatic) & SQ \\
\hline Shukla ${ }^{[17]}$ & 2008 & 24 & Retro & GBC & Visual \\
\hline
\end{tabular}

Note. Retro: Retrospective; Pro: Prospective; CC: Cholangiocarcinoma; GB: Gallbladder carcinoma; SQ: Semi-quantitative

Table 2. Summary of study findings

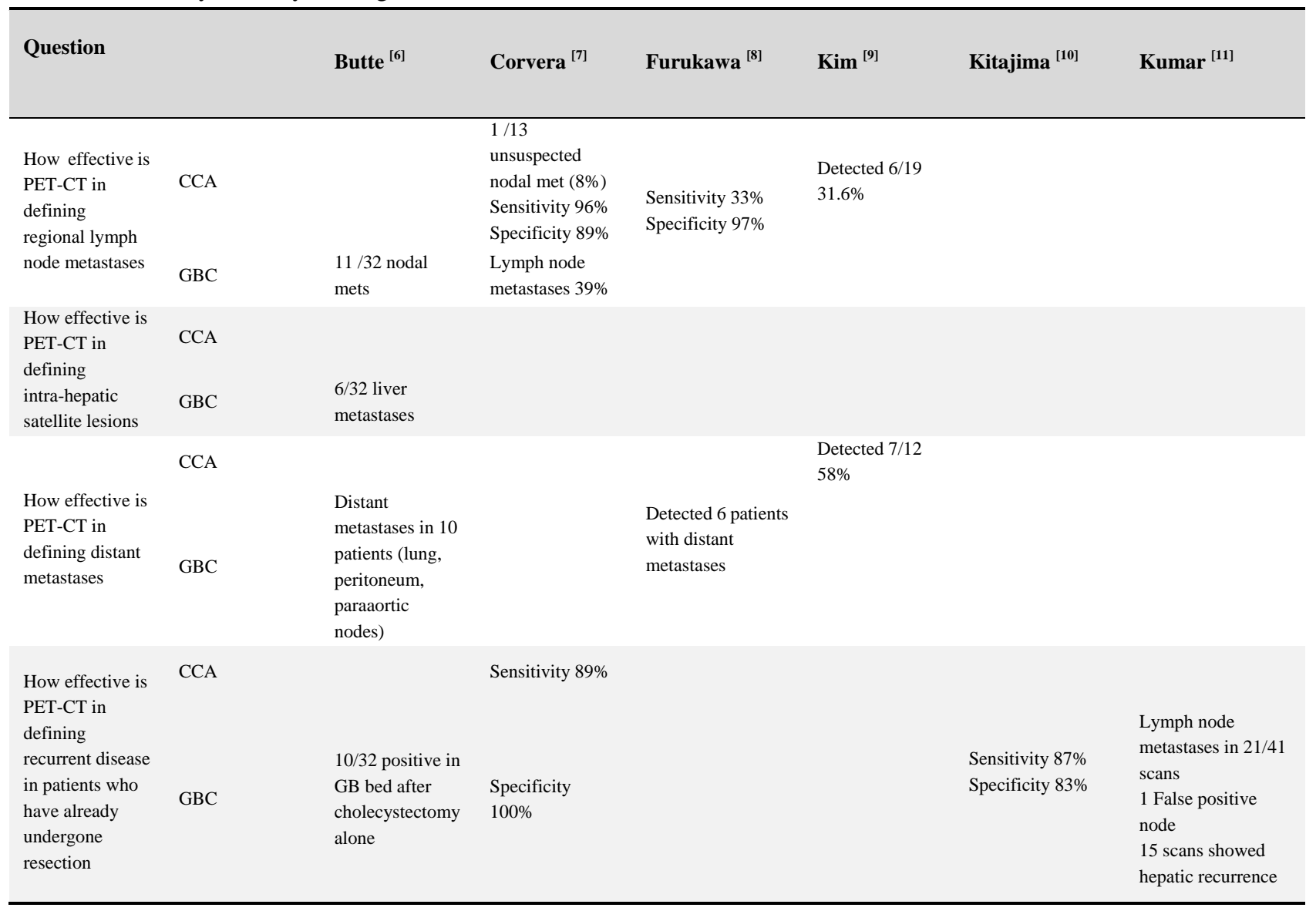


Table 2. (continued)

\begin{tabular}{|c|c|c|c|c|c|c|c|}
\hline Question & & Lee $2010^{[13]}$ & Lee $2011^{[12]}$ & $\mathbf{L i}^{[14]}$ & Ruys ${ }^{[15]}$ & Seo ${ }^{[16]}$ & Shukla ${ }^{[17]}$ \\
\hline $\begin{array}{l}\text { How effective } \\
\text { is PET-CT in } \\
\text { defining } \\
\text { regional lymph } \\
\text { node metastases }\end{array}$ & GBC & $\begin{array}{l}\text { Sensitivity } 82.1 \% \\
\text { Specificity } 95.3 \%\end{array}$ & Specificity 95\% & $\begin{array}{l}\text { Sensitivity } \\
41.7 \% \\
\text { Specificity } 80 \%\end{array}$ & $\begin{array}{l}\text { Sensitivity } \\
67 \% \\
\text { Specificity 67\% }\end{array}$ & $\begin{array}{l}7 / 28 \\
\text { Sensitivity } 43 \% \\
\text { Specificity } 100 \%\end{array}$ & $\begin{array}{l}\text { Specificity } \\
90 \%-100 \%\end{array}$ \\
\hline $\begin{array}{l}\text { How effective is } \\
\text { PET-CT in } \\
\text { defining } \\
\text { intra-hepatic } \\
\text { satellite lesions }\end{array}$ & GBC & & & & & & \\
\hline $\begin{array}{l}\text { How effective is } \\
\text { PET-CT in } \\
\text { defining distant } \\
\text { metastases }\end{array}$ & $\begin{array}{l}\text { CCA } \\
\text { GBC }\end{array}$ & & & & $\begin{array}{l}\text { Sensitivity 33\% } \\
\text { Specificity 96\% }\end{array}$ & & \\
\hline $\begin{array}{l}\text { How effective is } \\
\text { PET-CT in } \\
\text { defining } \\
\text { recurrent } \\
\text { disease in } \\
\text { patients who } \\
\text { have already } \\
\text { undergone } \\
\text { resection }\end{array}$ & GBC & & & & & & \\
\hline
\end{tabular}

\subsection{Gallbladder carcinoma}

Seven studies examined the role of PET-CT in gallbladder carcinoma ${ }^{[7,8,10,12,17,18]}$ though two studies ${ }^{[8,10]}$ did not report their results specifically. Butte et al ${ }^{[6]}$ investigated 32 patients with incidental gallbladder carcinoma following laparoscopic cholecystectomy. Thirteen patients had negative PET-CT results, 9 refused further resection with only one of the four patients resected having residual disease on operative exploration. PET-CT changed management in 12 out of 32 patients (38\%) demonstrating unexpected disseminated disease in 10 patients and localised resectable disease in 2 patients.

Corvera et al ${ }^{[7]}$ studied 31 patients with gallbladder cancer within their published series. They demonstrated a sensitivity of $86 \%$ and a specificity of $50 \%$ for detection of the primary tumour and a sensitivity of $87 \%$ and specificity of $89 \%$ for detection of nodal/ distant disease respectively. This resulted in a change in treatment for seven (23\%) patients. Shukla et al $^{[17]}$ evaluated the role of PET-CT in 24 patients with incidental gallbladder cancer prior to radical resection. They demonstrated that PET-CT predicted resectability with a sensitivity of $100 \%$ but was not significantly superior to conventional CT. PET-CT demonstrated residual disease with a sensitivity of $28.5 \%$ and with a specificity of $80.9 \%$. These results may have changed clinical management for two patients. Kumar et al ${ }^{[11]}$ evaluated the role of PET-CT in detecting recurrent gallbladder cancer in 49 patients. These investigators demonstrated a sensitivity and specificity of 97.6\% and 90\% respectively for detecting recurrent disease. PET/CT was shown to be more specific than conventional imaging (100\% vs. $50 \%)$ and would have resulted in a change in management for 5 (20\%) patients.

\subsection{Cholangiocarcinoma}

There were nine studies ${ }^{[7-10,12-16]}$ which evaluated the role of PET-CT in cholangiocarcinoma. Two studies reported their results as 'biliary' cancer with no further detail ${ }^{[8,13]}$. Other studies further stratified cholangiocarcinoma location into either intra-hepatic or extra-hepatic though did not present all endpoints of interest by subcategory ${ }^{[9,13]}$. 


\subsubsection{Extrahepatic cholangiocarcinoma}

Corvera et al ${ }^{[7]}$ studied 41 patients with extra-hepatic cholangiocarcinoma and demonstrated sensitivity and specificity of $69 \%$ and $67 \%$ respectively for detection of the primary tumour. Sensitivity and specificity for nodal and distant metastatic disease was $93 \%$ and $86 \%$ respectively and this led to a change in management for 8 (20\%) patients. The series by Kim et al ${ }^{[9]}$ included 87 patients with extra-hepatic cholangiocarcinoma and PET-CT demonstrated sensitivity and specificity of $81 \%$ and $79 \%$ respectively for detection of the primary tumour. There was no difference between PET-CT and conventional CT in the detection of the primary tumour but MRCP was shown to have significantly higher sensitivity than PET-CT.

Li et al ${ }^{[14]}$ investigated pre-operative staging of hilar cholangiocarcinoma in 17 patients with PET-CT and demonstrated a sensitivity of $59 \%$ for the detection of the primary tumour. Sensitivity and specificity for the detection of nodal disease was $42 \%$ and $80 \%$ respectively and $56 \%$ and $88 \%$ respectively for the detection of metastases.

Ruys et al ${ }^{[15]}$ conducted a study in 30 patients with hilar cholangiocarcinoma. The primary tumour was detected by PET-CT in 88\% of patients. Sensitivity and specificity for the detection of regional lymph node metastases were $67 \%$ and $68 \%$ and for distant metastases were 33\% and 96\% respectively. The authors reported a potential change in clinical management due to PET-CT findings in three (10\%) patients.

\subsubsection{I ntrahepatic cholangiocarcinoma}

The study by Corvera et al ${ }^{[7]}$ contained 20 patients with intrahepatic cholangiocarcinoma and PET-CT showed a sensitivity and specificity of $95 \%$ and $100 \%$ respectively for detection of the primary tumour. The sensitivity and specificity for detection of distant disease was $100 \%$ and $94 \%$ respectively resulting in a change in management for 7 (33\%) patients. Kim et al ${ }^{[9]}$ included 36 patients with intra-hepatic cholangiocarcinoma and PET-CT demonstrated sensitivity and specificity of $95 \%$ and $80 \%$ respectively for detection of the primary tumour. There was no difference between PET-CT and conventional CT or MRCP in detection of the primary tumour. Seo et al ${ }^{[16]}$ investigated PET-CT use in 27 patients with mass-forming intrahepatic cholangiocarcinoma. When PET-CT, CT and MRI were considered for the detection of nodal disease, the sensitivities were 43\%, 43\%, and 43\%, and the specificities were 100\%, 76\%, and 64\%, respectively. A high standardised uptake value on PET-CT was also shown to be independently associated with postoperative recurrence.

\subsubsection{Cholangiocarcinoma not otherwise specified}

For all patients in their series (intra-hepatic and extra-hepatic cholangiocarcinoma) Kim et al ${ }^{\text {[9] }}$ demonstrated that PET-CT had a higher specificity than CT for detection of regional lymph nodes (88\% vs. 65\%; $p<0.001)$ with no difference in sensitivity. PET-CT also had significantly higher sensitivity than CT for the detection of metastases $(58 \%$ vs. $0 \%$; $p=$ 0.02). PET-CT resulted in a change in management in 15 (16\%) patients.

\subsection{Biliary cancer not otherwise specified}

Furukawa et al ${ }^{[8]}$ investigated the role of PET-CT in 72 patients with either gallbladder cancer or extra-hepatic cholangiocarcinoma. PET-CT detected the primary cancer in 86\% of cases with CT diagnostic in 96\% of patients. PET-CT showed lower sensitivity (33\% vs. 57\%) and higher specificity (97\% vs. 79\%) than CT scan, although the values were not significantly different. Lee et al ${ }^{[13]}$ studied PET-CT in 99 patients with suspected gallbladder cancer and cholangiocarcinoma. There were no differences in sensitivity and specificity for the detection of primary tumour or lymph node metastases between PET-CT and CT. PET-CT was shown to have higher sensitivity than conventional CT in the detection of metastases (95\% vs. 63\%; $p=0.02)$. PET-CT changed management in six patients.

\subsection{Recurrent biliary cancer}

Corvera et al ${ }^{[7]}$ included 33 patients with suspected recurrent biliary cancer within their series. PET-CT was diagnostic in 25 (76\%) patients with a sensitivity and specificity of 89\% and 100\%. PET-CT changed clinical management in three (9\%) 
patients by correctly identifying disease not seen on conventional imaging. Kitajima et al ${ }^{[10]}$ studied the role of PET-CT in 50 patients with recurrent gallbladder cancer and cholangiocarcinoma. Amongst patients with suspected recurrence $(\mathrm{n}=$ 40), PET-CT showed sensitivity and specificity of $86 \%$ and $83 \%$ respectively. For the group of patients thought to be disease-free, PET-CT showed sensitivity and specificity of $100 \%$. The use of PET-CT resulted in a change in management for ten (20\%) out of 50 patients.

\section{Discussion}

This review was undertaken to clarify the effectiveness of PET-CT in primary or recurrent gallbladder cancer or cholangiocarcinoma. It was planned to perform a meta-analysis of the results of the systematic review but the heterogeneity of the publications, particularly those that included a number of cancer subtypes for analysis under the grouping of biliary cancer made this impossible to perform in a meaningful way. Consequently a narrative review was carried out. Despite the limitations of the available data as outlined below, a number of clinically relevant findings can be inferred.

PET-CT has a high specificity for regional lymph node metastases with most studies in cholangiocarcinoma reporting this between $89 \%$ and $100 \%{ }^{[7,8,13,16,17]}$. Ruys et al ${ }^{[15]}$ reported a lower specificity of $67 \%$ (12 of 18 positive nodes) respectively. These investigators emphasize that their results may have underestimated the effectiveness of PET-CT since three patients with positive scans did not have nodes assessed histologically because of tumour unresectability. Kim et al ${ }^{[9]}$ have emphasized that small tumour size and the growth pattern of cholangiocarcinomas, with small nests of cells embedded in a fibrous stroma, may compromise the accuracy of PET-CT in low volume deposits seen in regional lymph nodes. However, the high specificity emphasizes that a positive scan is a useful and accurate means of determining lymph node status preoperatively while a negative scan is less reassuring. Only Shukla et al ${ }^{[17]}$ formally assessed the efficacy of PET-CT in defining nodal metastases in different lymph node basins with specificities reported between $90 \%$ and $100 \%$ in nodes in the porta, retropancreatic, pericholedochal and para-aortic basins. There is reason to believe that similar results would be obtained for regional lymph node metastases in gallbladder cancer although only Butte et al ${ }^{[6]}$ report limited results for the use of PET-CT in these patients.

Minimal use of PET-CT has been made in the definition of satellite lesions in either cholancarcinoma or gallbladder cancer. Butte et al ${ }^{[6]}$ reported the effectiveness of PET-CT in defining liver metastases in patients with carcinoma of the gallbladder. All reported studies rely on standard cross-sectional imaging with triple phase CT scan or MRI to formally assess the liver for metastatic deposits ${ }^{[6,7]}$.

PET-CT has also been used in the detection of metastatic disease. Corvera et al ${ }^{[7]}$ found in patients with gallbladder cancer that PET-CT demonstrated metastatic disease in $23 \%$ in whom other imaging studies were normal and this included patients with extra-abdominal metastases (lung, mediastinal and bone) as well as intra-abdominal metastases (peritoneal and port site). Similar results were obtained in patients with cholangiocarcinoma in whom $37 \%$ had conclusive or suggestive evidence of distant metastatic disease on PET-CT.

The use of PET-CT in defining recurrent disease after treatment is an emerging indication as systemic therapies for patients with recurrent disease improve. Butte et al ${ }^{[6]}$ reported 10 of 32 patients with positive PET-CT scans with residual carcinoma in the gallbladder bed following initial treatment with laparoscopic cholecystectomy alone. Lee et al ${ }^{[13]}$ reported that PET-CT was useful in detecting lymph node metastases and hepatic recurrence following radical resection for gallbladder carcinoma. However Corvera et al ${ }^{[7]}$ emphasized that while PET-CT identified recurrent cancer in $76 \%$ of their patients, in all but three patients ( $9 \%$ of those evaluated) the recurrences were visible on standard cross-sectional imaging with CT or MRI ${ }^{[10]}$. 
This systematic review of the 12 publications has highlighted a number of issues within the PET-CT literature that make it difficult to obtain definitive answers to the four questions underpinning this review. Firstly, many studies group all cancers of the biliary tract together and present pooled data for cholangiocarcinoma (both intra and extra hepatic) and gallbladder cancer ${ }^{[8,13]}$. This probably reflects the low numbers of patients overall and a desire to optimise interpretation of imaging results. However, it is an oversimplification since the biology, paths of lymphatic spread and modes of surgical treatment are different for each cancer. A number of investigations have included patients presenting with gallbladder cancer following laparoscopic cholecystectomy and combine them with patients presenting with de novo disease ${ }^{[6]}$. Only the study of Shukla et al ${ }^{[17]}$ provides a breakdown of the anatomical sites of lymph node metastases. This is important since the presence of periportal node metastases does not mitigate against resection of either cholangiocarcinoma or gallbladder cancer but the presence of nodal metastases in the common hepatic or celiac nodal basins would be a contraindication. The reviewed studies also differ in the descriptive methodology used to describe results. Most use sensitivity and specificity to describe the accuracy of PET-CT in various settings ${ }^{[7]}$ although others simply describe PET positivity rates and numbers of patients in whom PET-CT findings resulted in a change in management ${ }^{[9]}$. Because of the retrospective and observational nature of all the investigations a significant number of patients did not undergo resection and consequently there was no histological confirmation of PET-CT findings ${ }^{[8,9]}$.

Despite these caveats, the following recommendations for the current use of PET-CT in the assessment of cholangiocarcinoma and gallbladder cancer can be made.

1) PET-CT has a low sensitivity for regional lymph node metastases from cholangiocarcinoma and gallbladder cancer but a high specificity. There is a role for PET-CT scan to investigate abnormal appearing lymph nodes seen on standard cross-sectional imaging if a positive finding will change management. Based on a single study PET-CT appears to be effective in assessing all nodal basins related to the liver, bile ducts and gallbladder.

2) PET-CT has no role in the assessment of hepatic satellite lesions.

3) PET-CT is useful in defining metastatic disease but predominantly in patients with abnormalities in standard cross-sectional imaging. PET-CT should be reserved to assess specific areas of concern defined on CT and/or MRI.

4) For patients with potentially recurrent disease, most recurrences (either intra or extra-abdominal) will be visible on CT or MRI. PET-CT should be reserved for the investigation of specific abnormalities defined with CT or MRI

\section{References}

[1] Khan SA, Thomas HC, Davidson BR, et al. Cholangiocarcinoma. Lancet. 2005; 366: 1303-14.

http://dx.doi.org/10.1016/S0140-6736(05)67530-7

[2] Gourgiotis S, Kocher HM, Solani L, et al. Gallbladder cancer. Am J Surg. 2008; 196: 252-64.

http://dx.doi.org/10.1016/j.amjsurg.2007.11.011

[3] Oshiro Y, Takahashi K, Sasaki R, et al. Adjuvant surgery for advanced extrahepatic cholangiocarcinoma. World J Gastroenterol. 2013; 19: 6934-38. http://dx.doi.org/10.3748/wjg.v19.i40.6934

[4] Cameron K, Golan S, Simpson W, et al. Recurrent pancreatic carcinoma and cholangiocarcinoma: 18F- Fluorodeoxyglucose positron emission tomography/computed tomography (PET/CT). Abdominal imaging. 2011; 36: 463-71.

http://dx.doi.org/10.1007/s00261-011-9729-6

[5] Prisma Statement. Available from: www.prisma-statement.org Accessed May 12, 20142012.

[6] Butte JM, Redondo F, Waugh E, et al. The role of PET-CT in patients with incidental gallbladder cancer. HPB. 2009 ; $11: 585-591$. http://dx.doi.org/10.1111/j.1477-2574.2009.00104.x

[7] Corvera CU, Blumgart LH, Akhurst T, et al. 18F-fluorodeoxyglucose positron emission tomography influences managment decisions in [patients with biliary cancer. J Am Coll Surg. 2008; 206: 57-65. http://dx.doi.org/10.1016/j.jamcollsurg.2007.07.002 
[8] Furukawa H, Ikuma H, Asakura-Yukoe K, et al. Preoperative staging of biliary carcinoma using 18F-fluorodeoxyglucose PET: prospective comparison with PET + CT, MDCT and histopathology. Eur Radiol. 2008; 18: 2841-2847. http://dx.doi.org/10.1007/s00330-008-1062-2

[9] Kim JY, Kim M-H, Lee TY, et al. Clinical role of 18FDG PET-CT in suspected and potentially operable cholangiocarcinoma: A prospective study compared with conventional imaging. Am J Gastroenterol. 2008; 103: 1145-1151. http://dx.doi.org/10.1111/j.1572-0241.2007.01710.x

[10] Kitajima K, Murakami K, Kanegae K, et al. Clinical impact of whole body FDG-PET for recurrent biliary cancer: a multicenter study. Ann Nucl Med. 2009; 23: 709-715. http://dx.doi.org/10.1007/s12149-009-0297-6

[11] Kumar R, Sharma P, Kumari A, et al. Role of 18F-FDG PET/CT in detecting recurrent gallbladder carcinoma. Clin Nuclear Med. 2012; 37: 431-435. http://dx.doi.org/10.1097/RLU.0b013e31824d24c4

[12] Lee YG, Han SW, Oh DY, et al. Diagnostic performance of contrast enhanced CT 18FDG PET/CT in suspicious recurrence of biliary tract cancer after curative resection. BMC Cancer. 2011; 11: 188-195. http://dx.doi.org/10.1186/1471-2407-11-188

[13] Lee SW, Kim HJ, Park JH, et al. Clinical usefulness of 18F-FDG PET-CT for patients with gallbladder cancer and cholangiocarcinoma. J Gastroenterol. 2010; 45: 560-566. http://dx.doi.org/10.1007/s00535-009-0188-6

[14] Li J, Kuehl H, Grabellus F, et al. Preoperative assessment of hilar cholangiocarcinoma by dual-modality PET/CT. J Surgical Oncology. 2008; 98: 438-443. http://dx.doi.org/10.1002/jso.21136

[15] Ruys AT, Bennink RJ, van Westreenen HL, et al. FDG-positron emission tomography/computed tomography and standardized uptake value in the primary diagnosis and staging of hilar cholangiocarcinoma. HPB. 2011; 13: 256-262. http://dx.doi.org/10.1111/j.1477-2574.2010.00280.x

[16] Seo S, Hatano H, Higashi T, et al. Fluorine-18 fluorodeoxyglucose positron emission tomography predicts lymph node metastases, P-glycoprotein expression, and recurrence after resection in mass-forming intrahepatic cholangiocarcinoma. Surgery. 2008; 143: 769-77. http://dx.doi.org/10.1016/j.surg.2008.01.010

[17] Shukla PJ, Barreto SG, Arya S, et al. Does PET-CT scan have a role prior to radical re-resection for incidental gallbladder cancer? HPB. 2008; 10: 439-45. http://dx.doi.org/10.1080/13651820802286910

[18] Butte JM, Matsuo K, Gonen M, et al. Gallbladder cancer: Differences in presentation, surgical treatment and survival in patients treated at centers in three countries. J Am Coll Surg. 2010; 212: 50-61. http://dx.doi.org/10.1016/j.jamcollsurg.2010.09.009 